
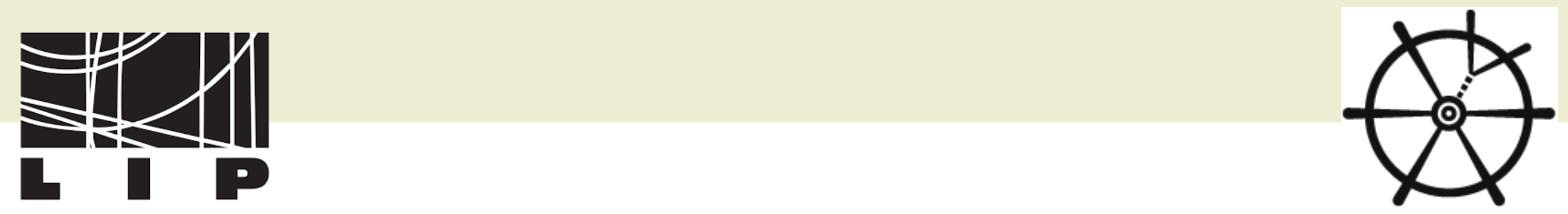

SHiP

Search for Hidden Particles

\title{
The SHiP timing detector based on MRPC
}

Alberto Blanco ${ }^{1}$, Celso Franco ${ }^{1}$, Custodio Loureiro ${ }^{2}$, Filomena Clemencio ${ }^{3}$, Guilherme Soares ${ }^{1}$, João Saraiva ${ }^{1}$, Luis Lopes ${ }^{1}$, Nuno Leonardo ${ }^{1}$, Paulo Fonte ${ }^{1}$

${ }^{1}$ LIP, Laboratório de Instrumentação e Física Experimental de Partículas

${ }^{2}$ LIBPhys, Departamento de Física, Universidade de Coimbra

${ }^{3}$ Escola Superior de Saúde do Politécnico do Porto

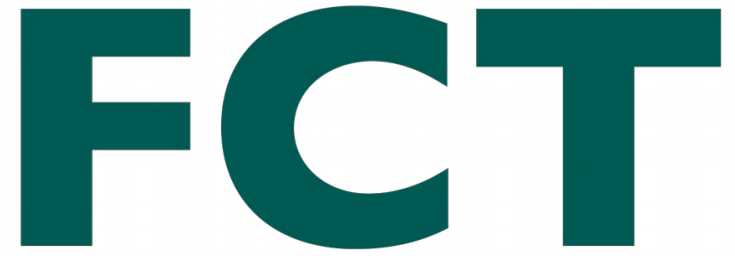

Fundação para a Ciência e a Tecnologia MINISTÉRIO DA CIÊNCIA, TECNOLOGIA E ENSINO SUPERIOR

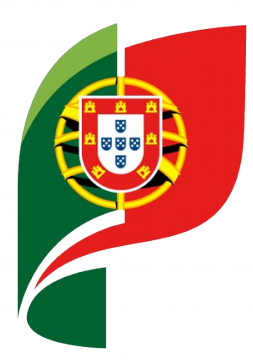

REPÚBLICA PORTUGUESA 
- The SHiP experiment.

- Timing detector for SHiP based on MRPCs.

- Test beam result at CERN.

- Conclusions. 


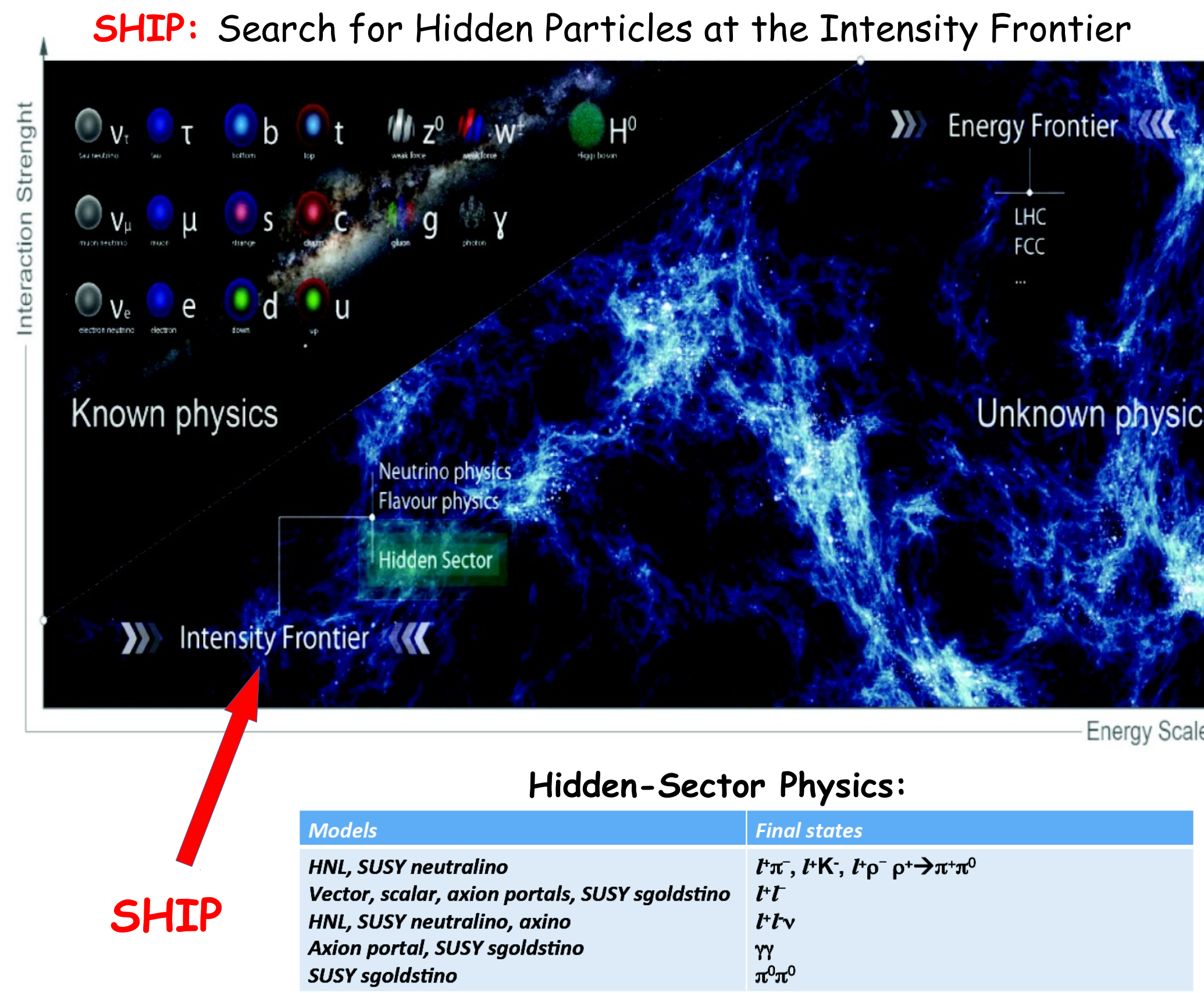




\section{Search for Heavy Neutral Leptons (HNLs)}

Standard Model

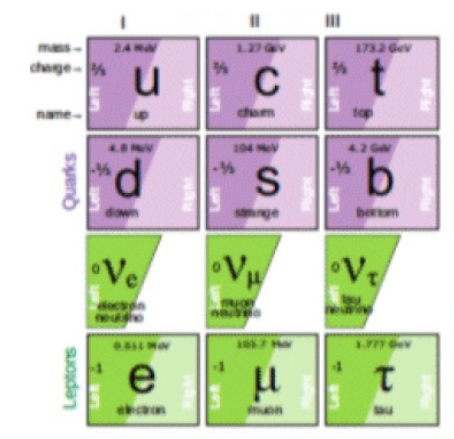

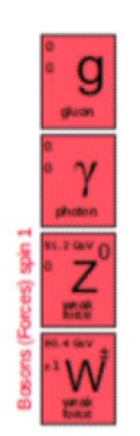

$N=$ Heavy Neutral Lepton

- Role of $\mathbf{N}_{1}$ with mass of few $\mathrm{KeV}$ : $\underline{\text { dark }}$ matter

- Role of $\mathbf{N}_{2}, \mathbf{N}_{3}$ with mass in $100 \mathrm{MeV}$ to $2 \mathrm{GeV}$ region: "give" masses to neutrinos and produce baryon asymmetry of the Universe

\section{Tau neutrino $\left(\tau_{v}\right)$ physics}

$v$ interaction rates for 5 years of nominal operation $\left(2 \times 10^{20}\right.$ p.o.t)

\begin{tabular}{|c|c|c|}
\hline & $\Phi$ & $<\mathrm{E}\rangle(\mathrm{GeV})$ \\
\hline$v_{\mu}$ & $1.7 \times 10^{6}$ & 29 \\
\hline $\mathbf{v}_{\mathrm{e}}$ & $2.5 \times 10^{5}$ & 46 \\
\hline $\mathbf{V}_{\mathbf{\tau}}$ & $7.6 \times 10^{3}$ & 59 \\
\hline Anti- $v_{\mu}$ & $6.7 \times 10^{5}$ & 28 \\
\hline Anti-v & $9.0 \times 10^{4}$ & 46 \\
\hline Anti- $v_{\tau}$ & $3.9 \times 10^{3}$ & 58 \\
\hline
\end{tabular}

First experimental measurement of anti- $v_{\tau}$ interactions: 
Neutrino DIS: structure functions and strange quark nucleon content

$$
\begin{aligned}
\frac{d^{2} \sigma^{\nu(\bar{\nu})}}{d x d y} & =\frac{G_{F}^{2} M E_{\nu}}{\pi\left(1+Q^{2} / M_{W}^{2}\right)^{2}}\left(\left(y^{2} x+\frac{m_{\tau}^{2} y}{2 E_{\nu} M}\right) F_{1}+\left[\left(1-\frac{m_{\tau}^{2}}{4 E_{\nu}^{2}}\right)-\left(1+\frac{M x}{2 E_{\nu}}\right)\right] F_{2}\right. \\
& \left. \pm\left[x y\left(1-\frac{y}{2}\right)-\frac{m_{\tau}^{2} y}{4 E_{\nu} M}\right] F_{3}+\frac{m_{\tau}^{2}\left(m_{\tau}^{2}+Q^{2}\right.}{4 E_{\nu}^{2} M^{2} x}-\frac{m_{\tau}^{2}}{E_{\nu} M} F_{5}\right)
\end{aligned}
$$

First evaluation of $\mathbf{F}_{4}$ and $\mathbf{F}_{5}$ (not accessible with $v_{e}$ and $\left.v_{\mu}\right)$

Light Dark Matter (LDM) searches 
- Modules composed of two $6(0.3 \mathrm{~mm})$ gaps sealed glass stacks (SGS).

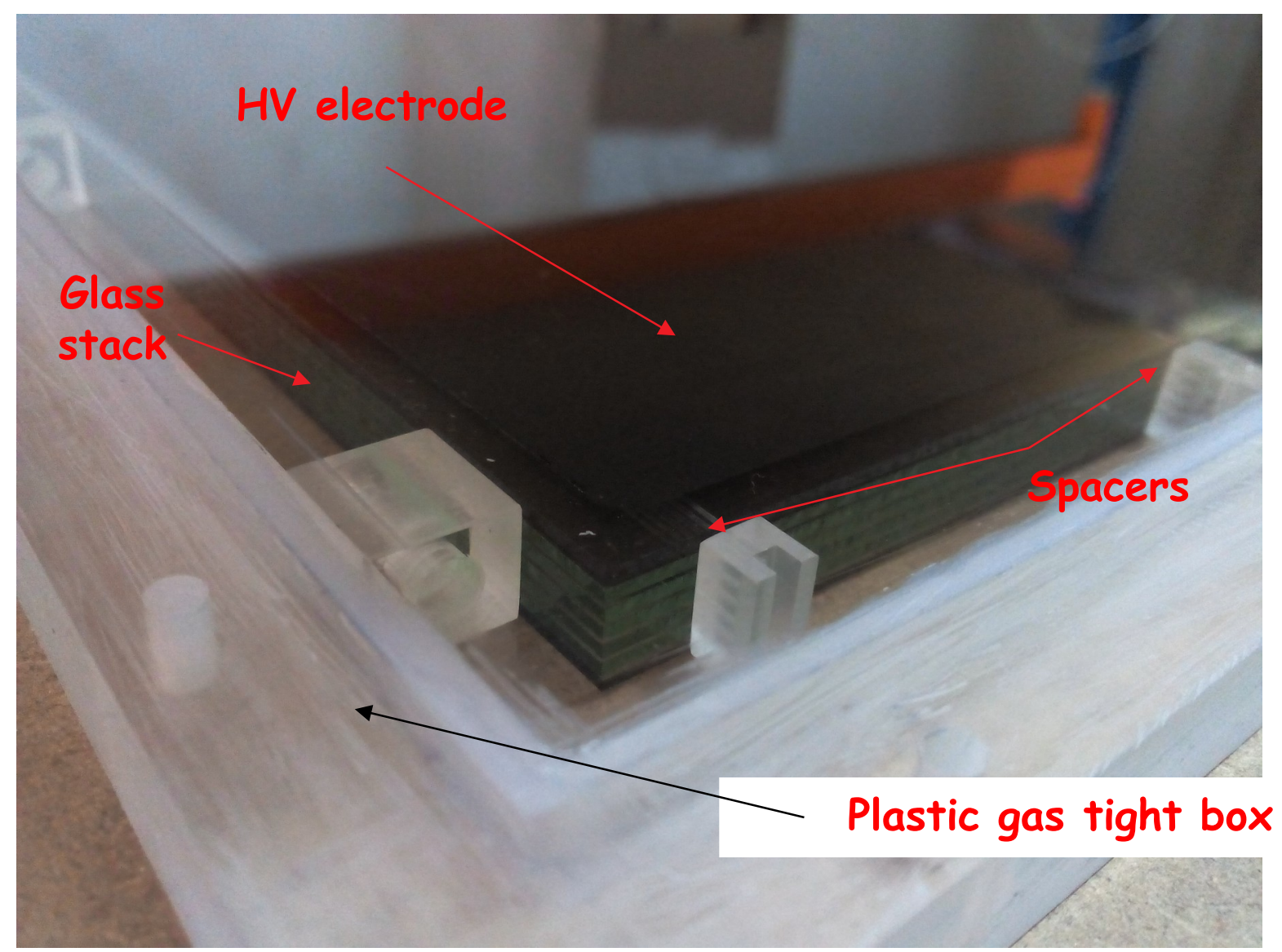

A sealed glass stack contains the glass and HV electrodes enclosed in a plastic gas tight box with feed-throughs for gas and High Voltage.

Easy to build completely gas tight, no gas leaks, robust. Low gas consumption

Decouples the gas and HV from the rest, specifically readout electrodes

In competition with a scintillator based approach 
- Modules composed of two $6(0.3 \mathrm{~mm})$ gaps sealed glass stacks (SGS).

- Strips $37 \mathrm{~mm}$ width (placed in the middle of two SGS) readout in both sides.

- Active area of $1600 \times 1200 \mathrm{~mm}^{2}=1,9 \mathrm{~m}^{2}$.

- Good time precision, < 60 ps $\sigma$.

- Good efficiency, > $98 \%$.

- Easy to build.

MRPC top View

6 gaps RPC

Strip readout glass stack

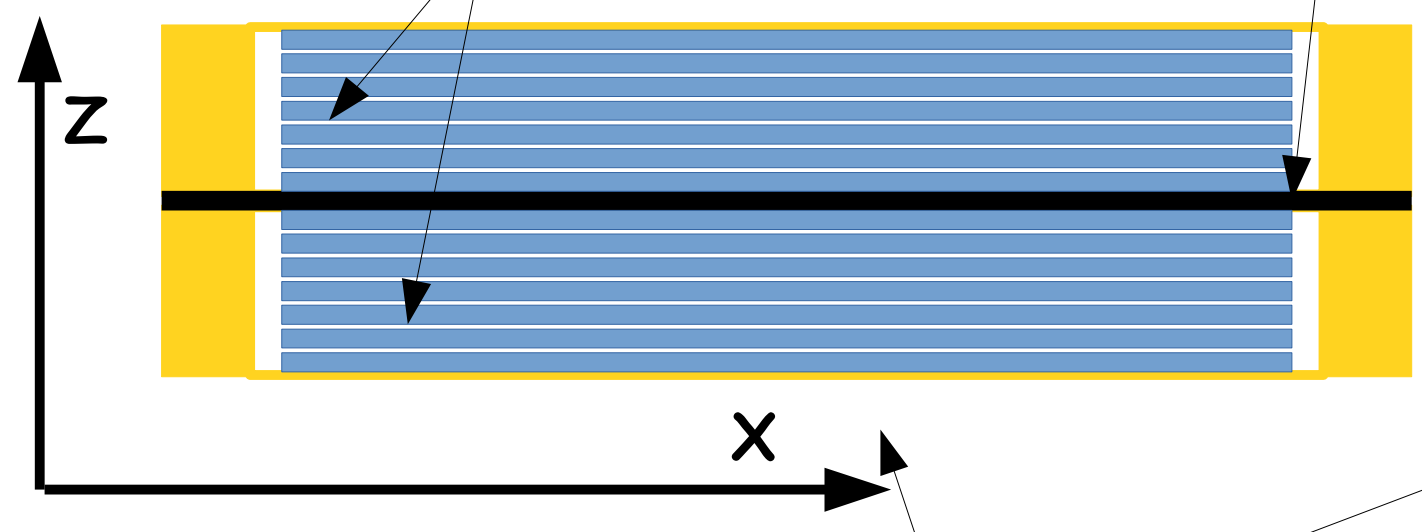

Module

MRPC cross-section

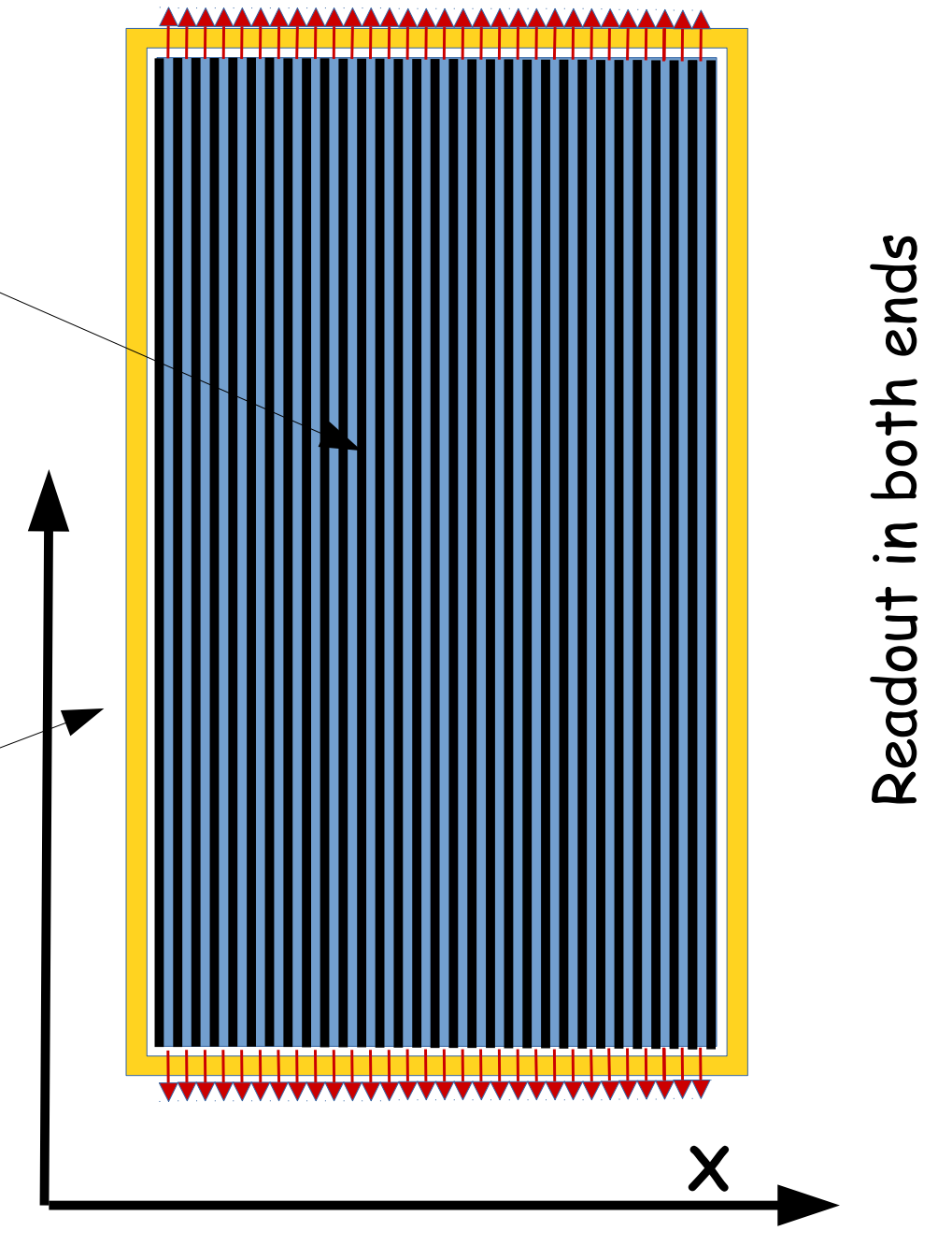


- Modules composed of two 6 gaps sealed glass stacks (SGS).

- Strips $37 \mathrm{~mm}$ width (placed in the middle of two SGS) readout in both sides.

- Active area of $1600 \times 1200 \mathrm{~mm}^{2}=1,9 \mathrm{~m}^{2}$.

- Good time precision, < 60 ps $\sigma$.

- Good efficiency, > $98 \%$.

- Easy to build.

\section{Side}

view
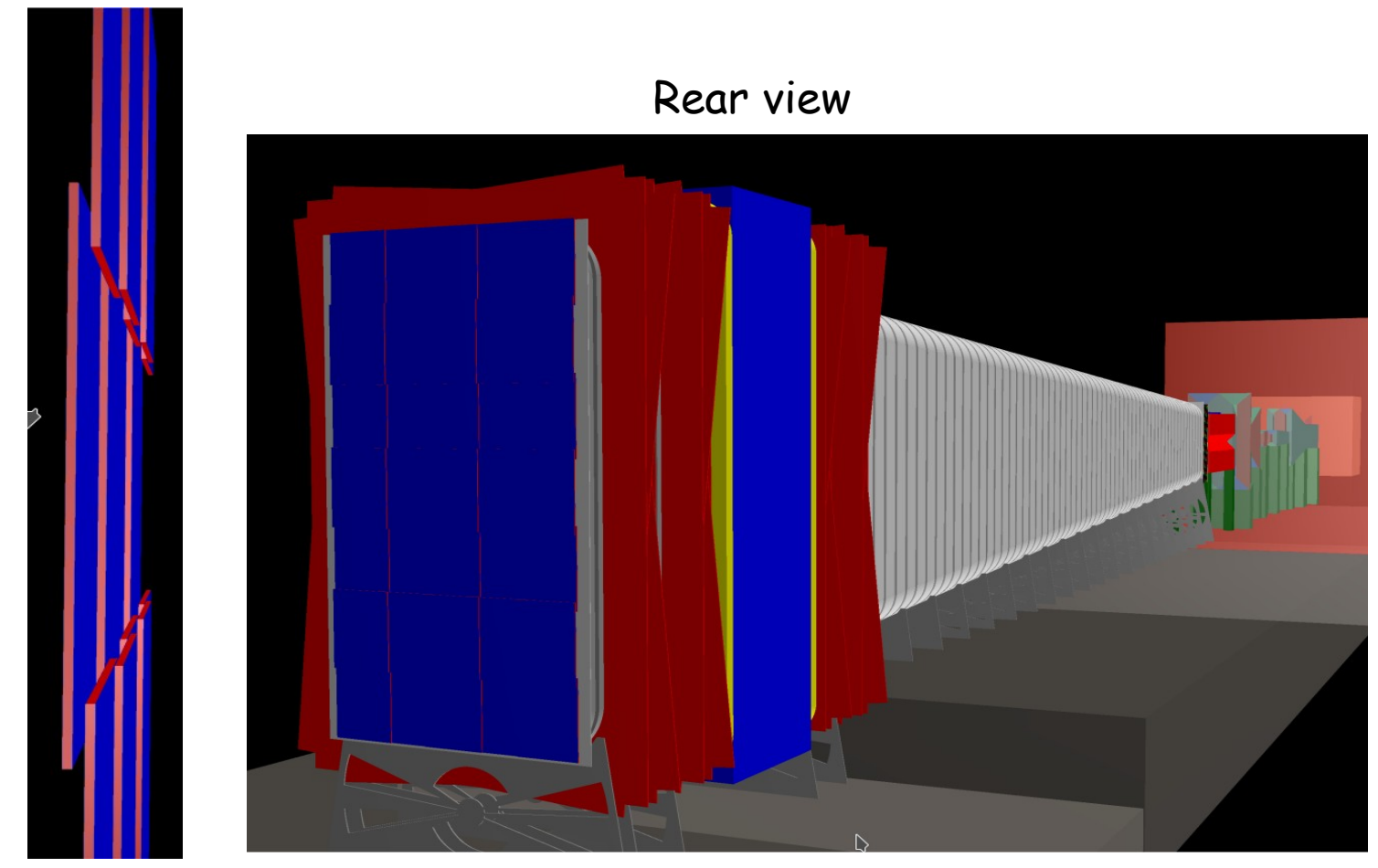

Area to be covered $10 \times 5 \mathrm{~m}^{2}$

$\Rightarrow 35$ MRPC modules with overlap

$\Rightarrow 35$ modules $\times 64$ channels/module

$=2240$ channels.

MRPC timing detector implementation

in Fair SHiP (SHiP experiment software). 
Test beam. Setup.

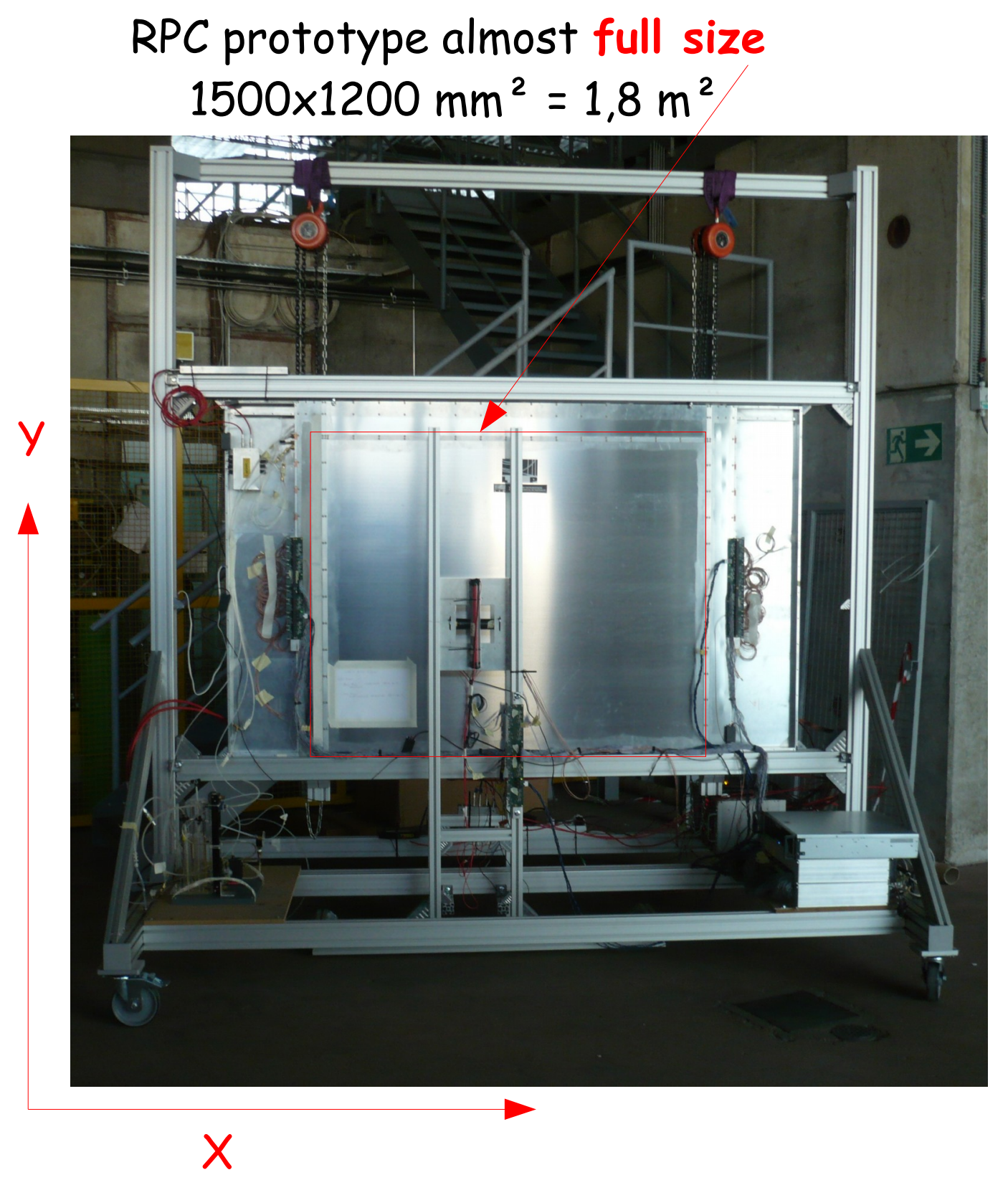


RPC prototype almost full size $1500 \times 1200 \mathrm{~mm}^{2}=1,8 \mathrm{~m}^{2}$

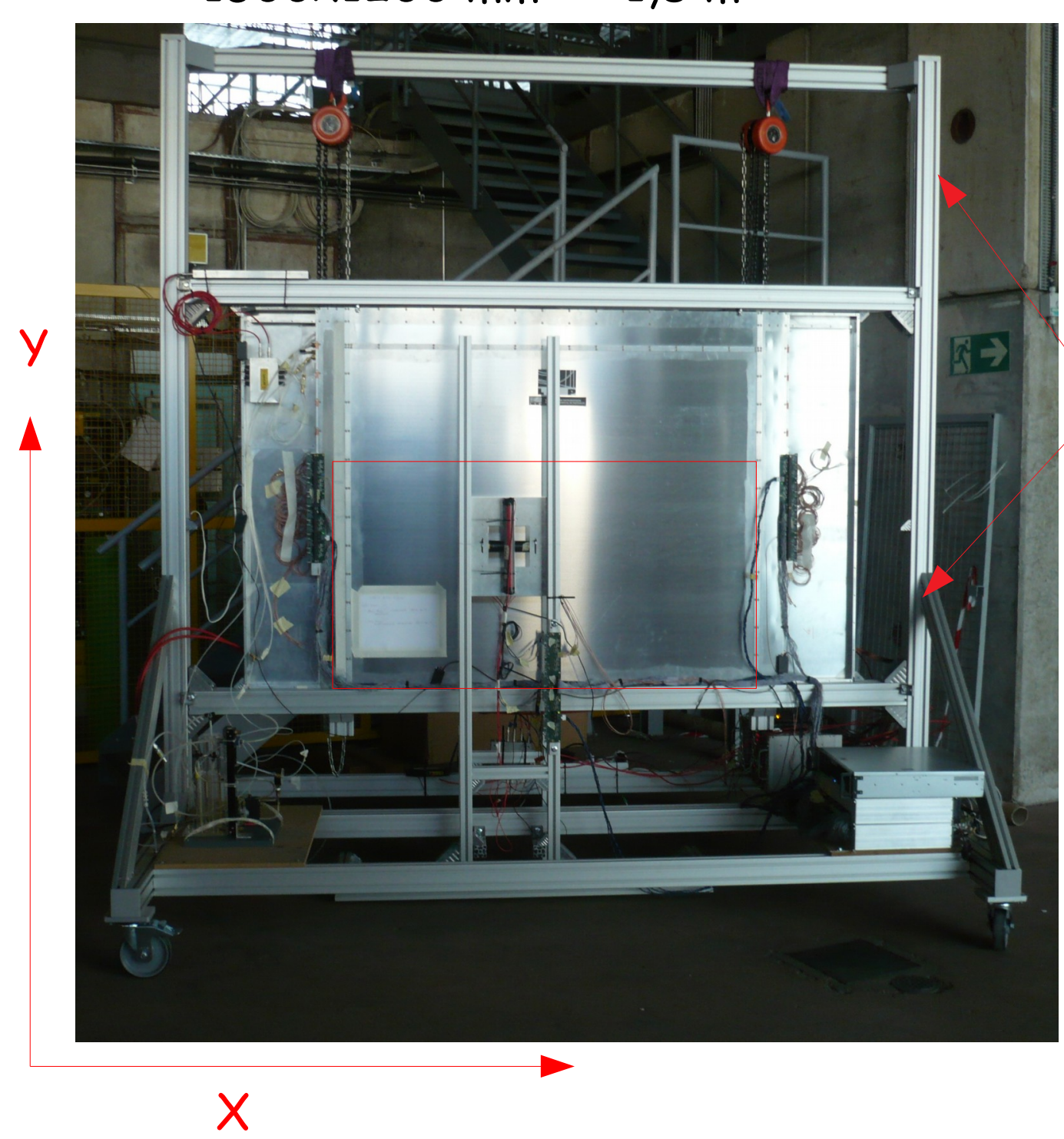

Due to limitations on the mechanical structure that holds the RPC only $2 / 3$ of the active area can be scanned 
Test beam. Setup.

FEE borrowed from HADES-RPC TOF

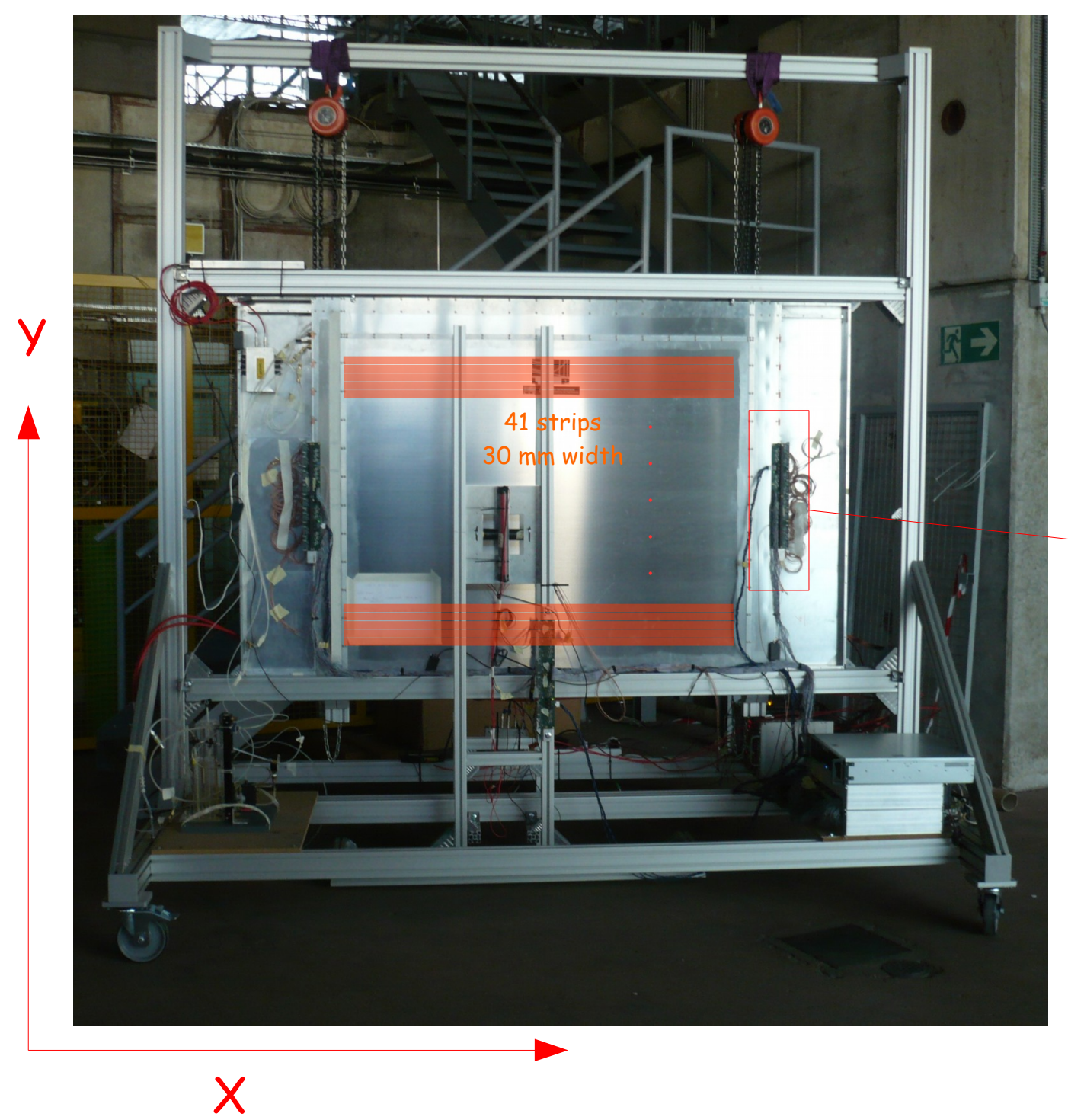

FEE, time $\left(\sigma_{t} \sim 30 \mathrm{ps}\right)$ and charge measurement in one single channel. Strips are readout in both sides

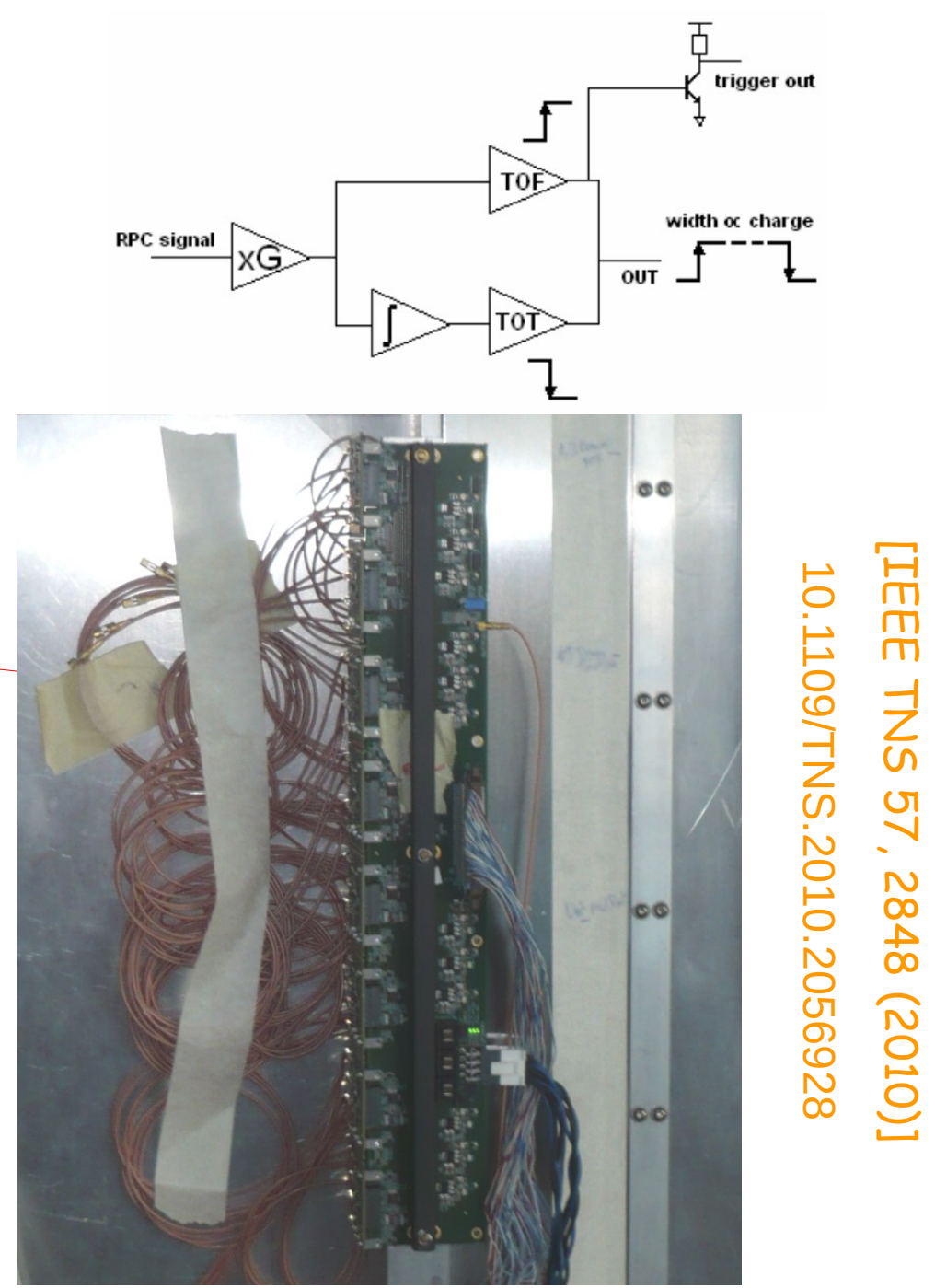

32 channels each side 
Test beam. Setup.

DAQ borrowed from HADES DAQ.

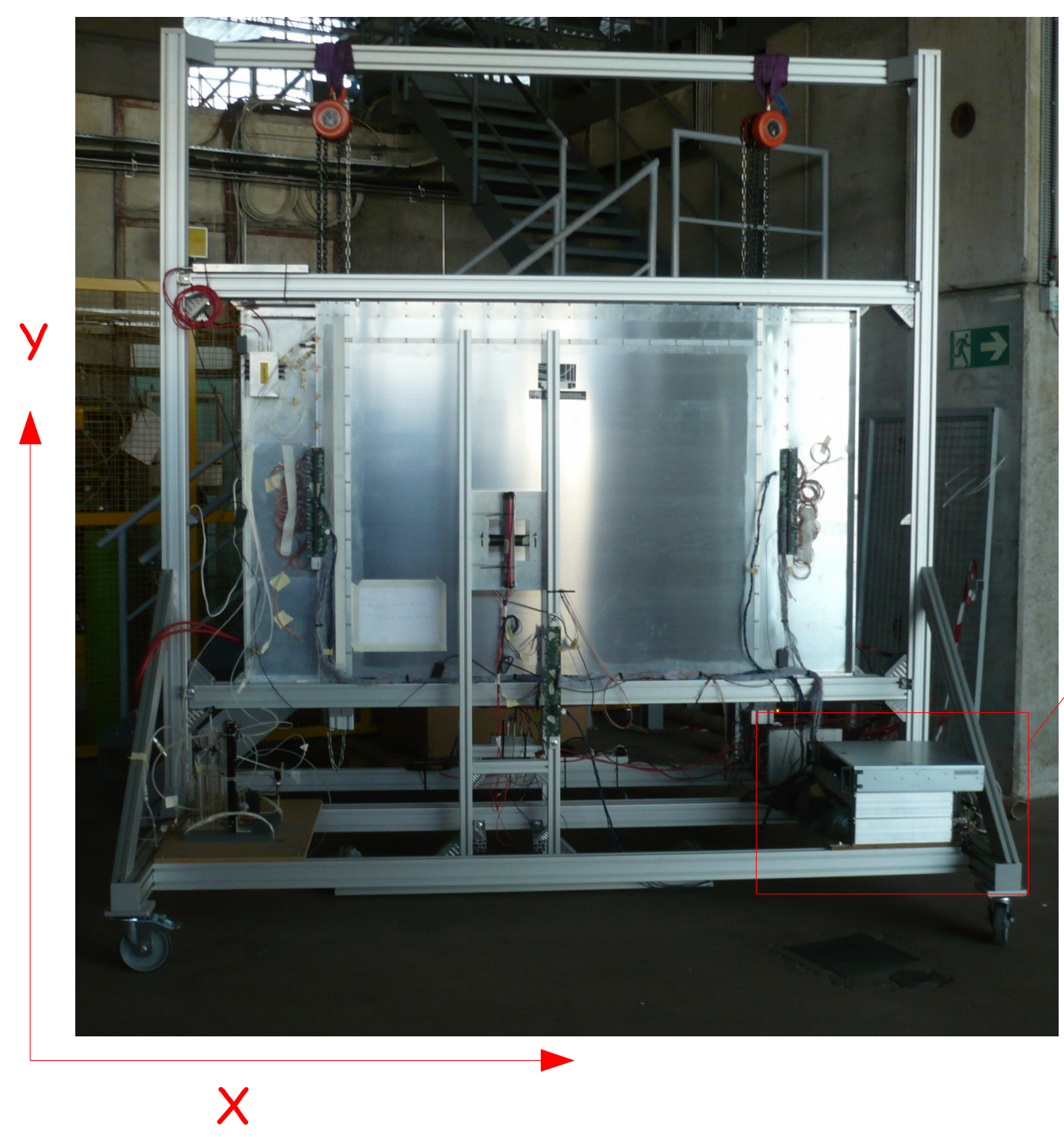

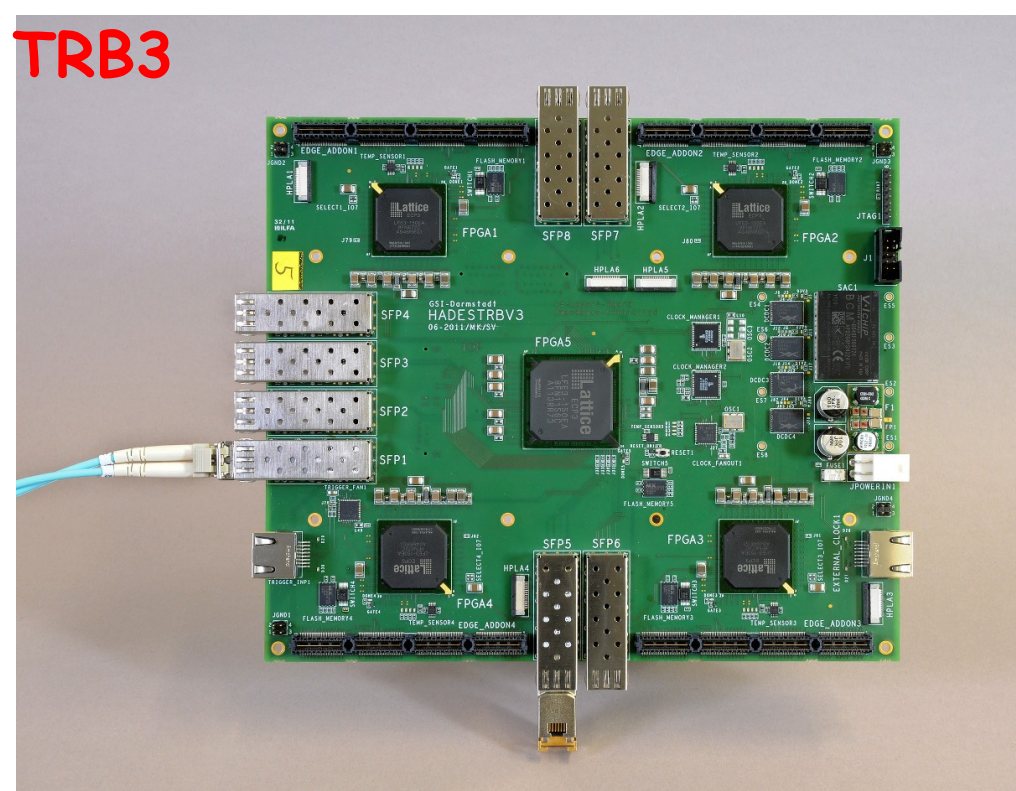

One central FPGA with trigger management capabilities plus

- $4 \times 32$ Multi-hit TDC Time precision $<20$ ps And much more, ADCs .... 
Fast timing scintillators telescope 2 in front and 2 in the rear. 30-40 ps $\sigma$ in beam. Used as a reference

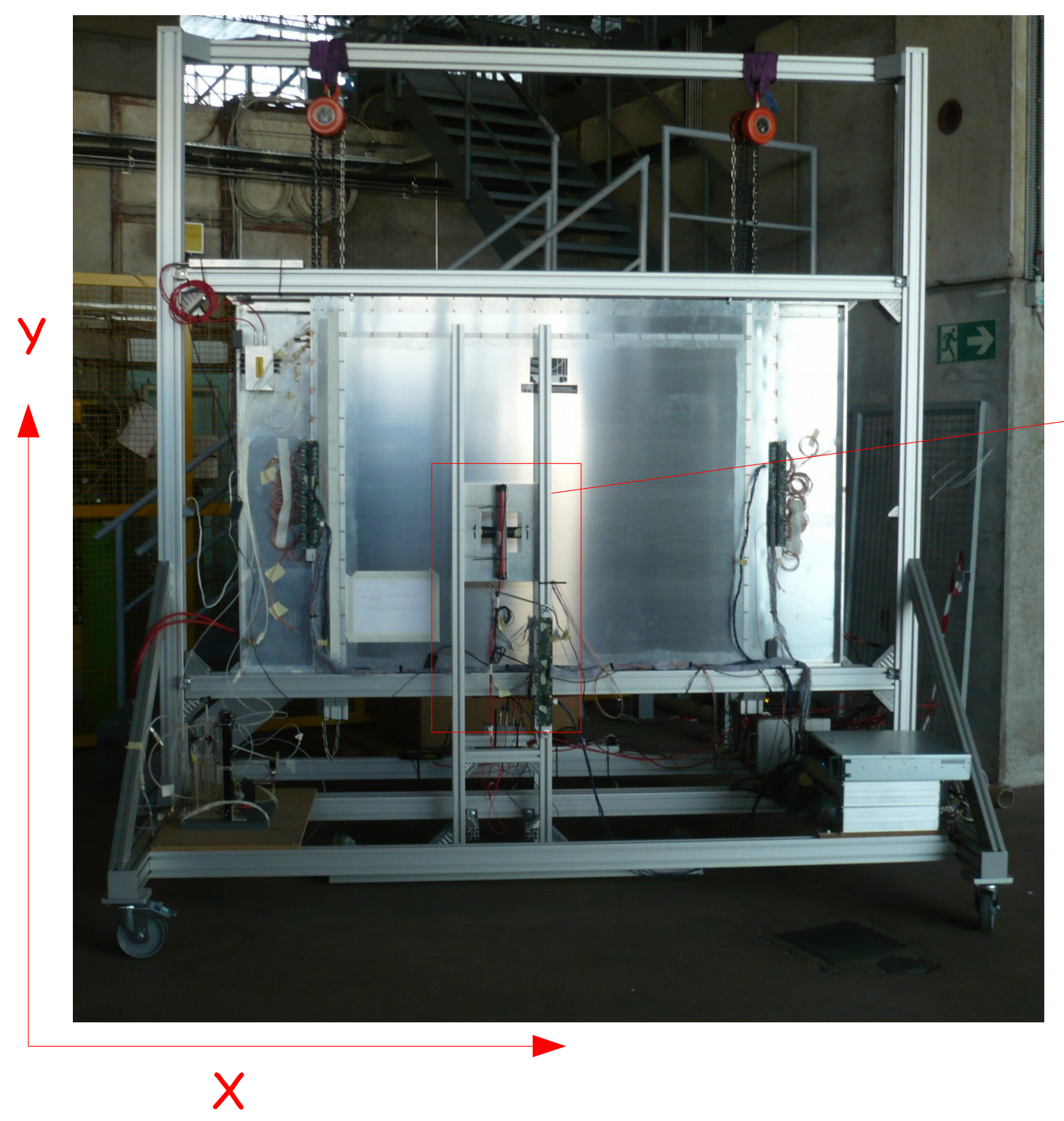

The SHiP timing detector based on MRPC

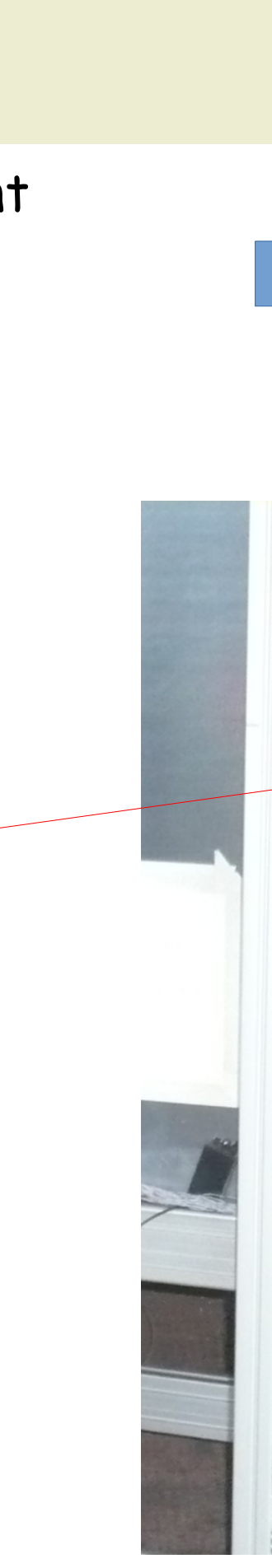

Sc1

$\mathrm{Sc2}$ RPC

$\mathrm{Sc} 3$

$5 c 4$
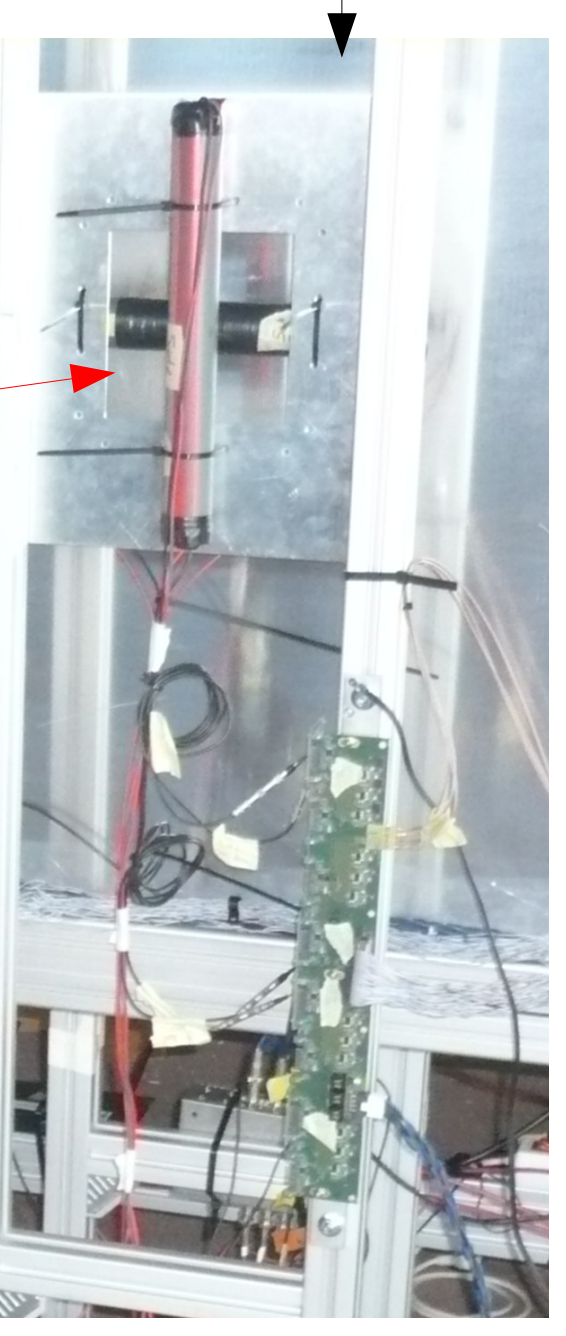

RPC Workshop 2020 Roma 10-14 February 
Test beam. Setup.

\section{Ancillary systems}

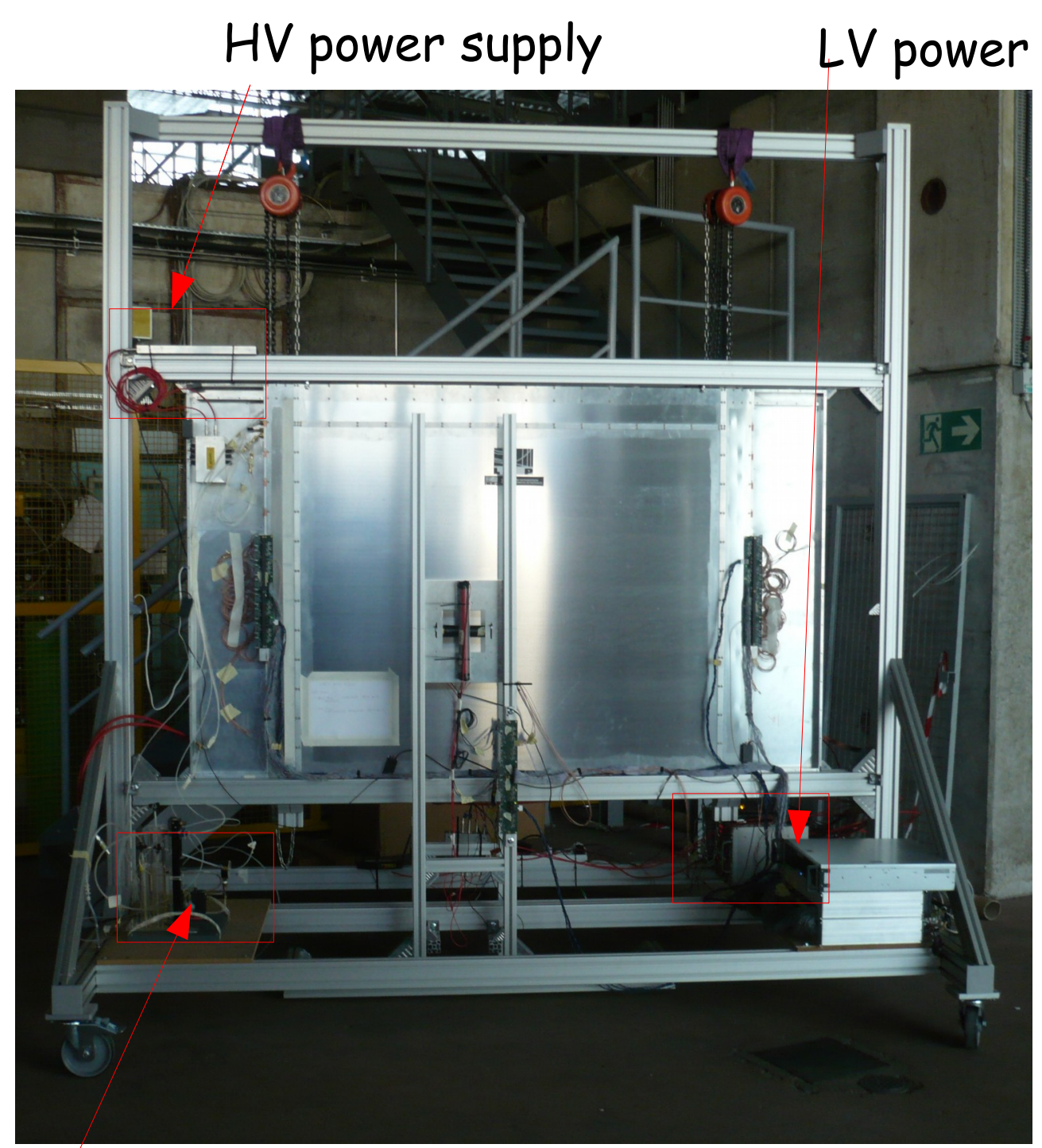

Gas system, $97.5 \% \mathrm{C}_{2} \mathrm{H}_{2} \mathrm{~F}_{4}+2,5 \% \mathrm{SF}_{6} @ 50 \mathrm{cc} / \mathrm{min}$ 


\section{All measurements made with negative $8 \mathrm{GeV}$ pions}

Efficiency

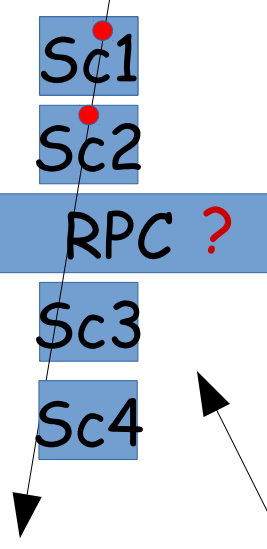

Timing precision

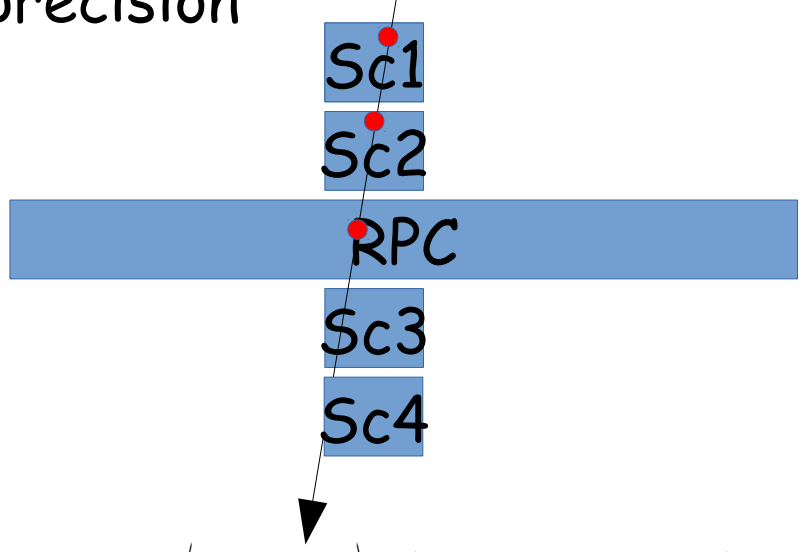

$\operatorname{sic} 2$ RPC

$\mathrm{Sc} 3$ Sc4
(

Preliminary analysis only include two scintillators

Time from RPC and scintillators are corrected by charge. Walk correction.
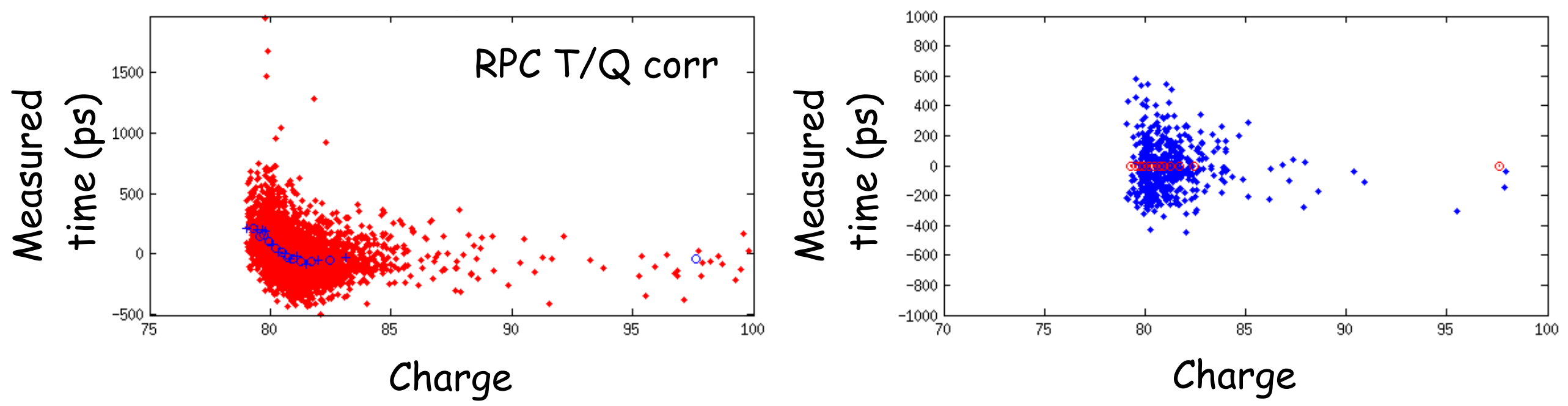


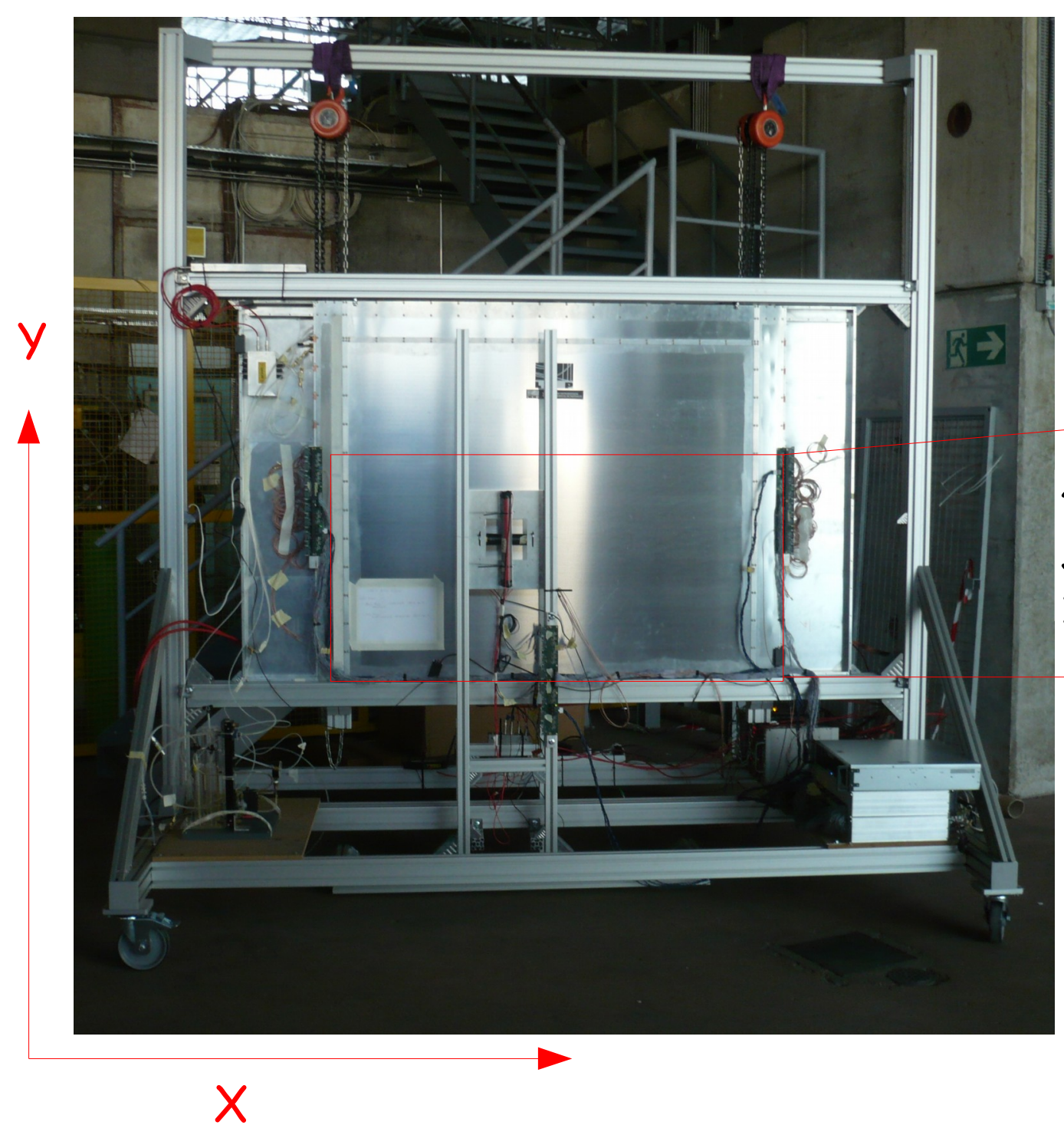

Fine horizontal scan

\begin{tabular}{c|c} 
& $\begin{array}{c}\text { Two full } \\
\text { Fine vertical scan }\end{array}$ \\
horizontal scans
\end{tabular}

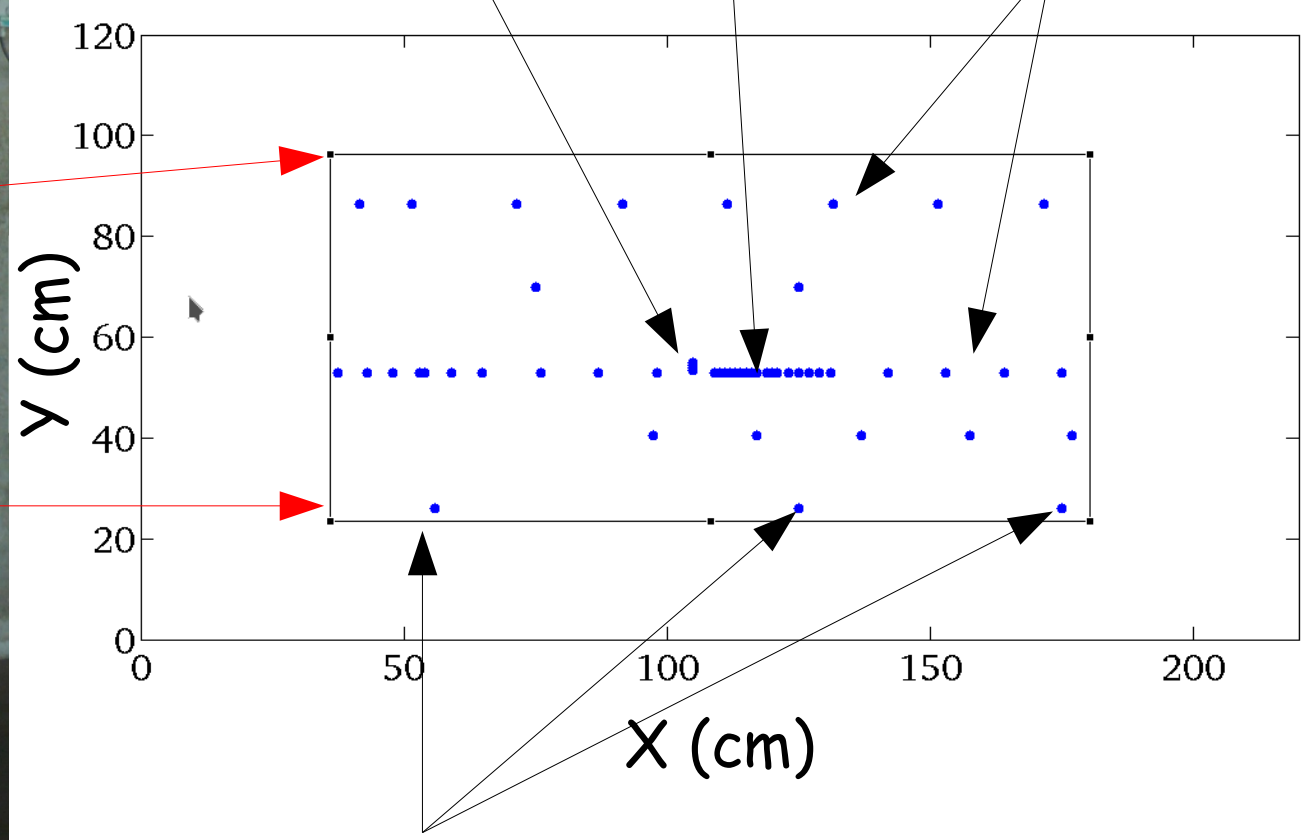

Edges scanned 
Test beam. Efficiency and timing vs X, Y.

No noticeable dependence with position
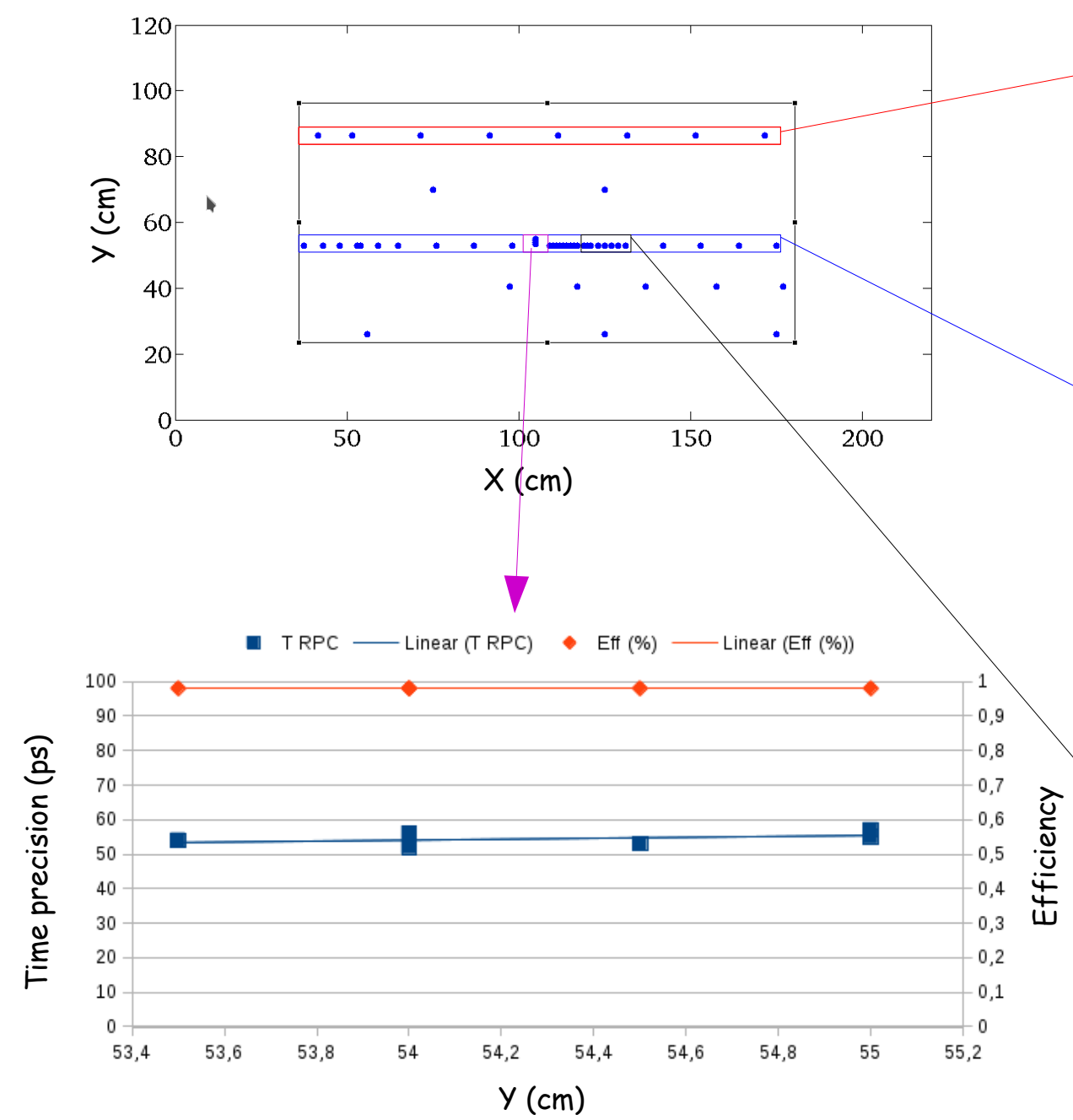

- Eff (\%) — Linear (Eff (\%)) - T RPC — Linear (T RPC)
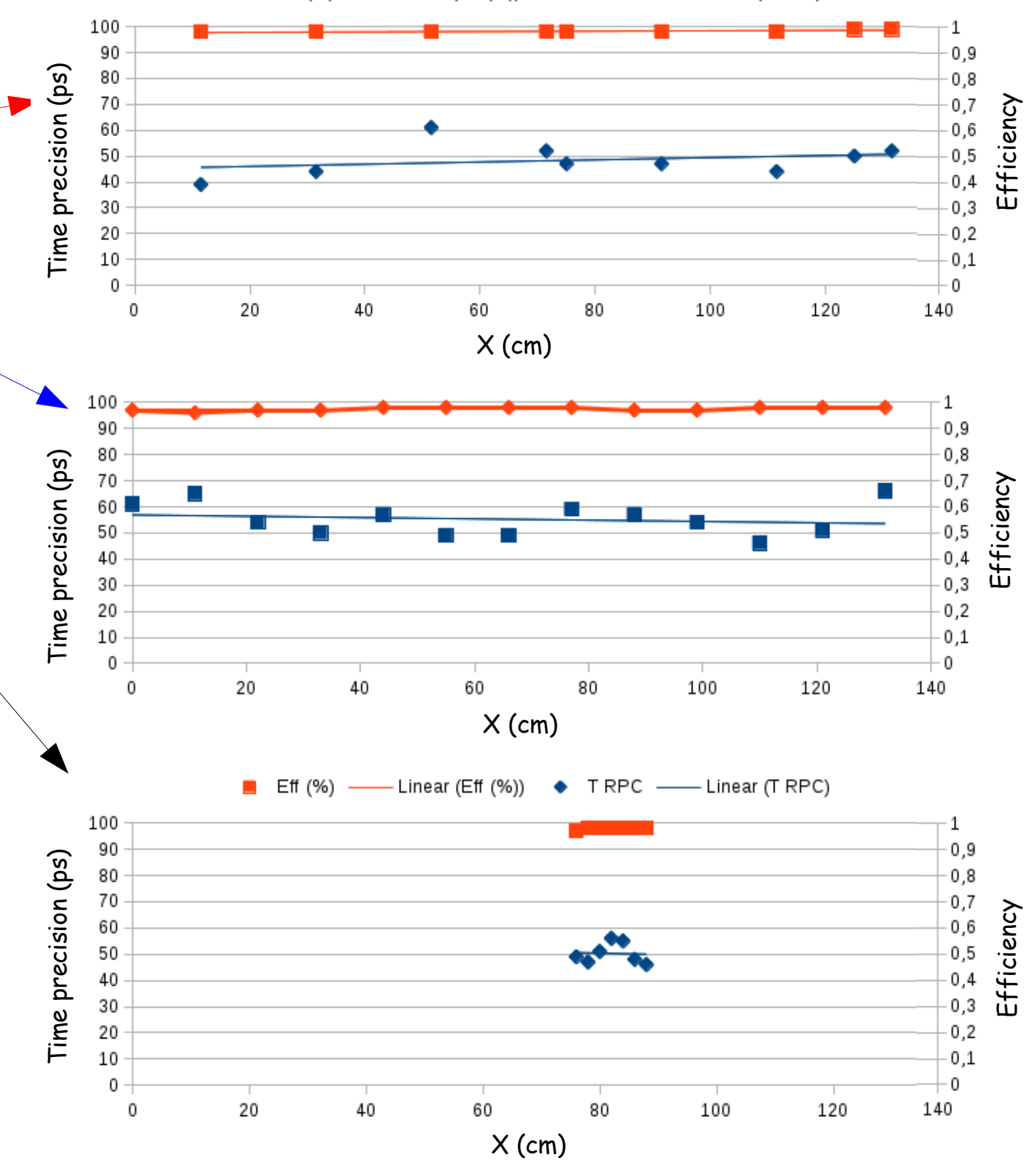
All positions

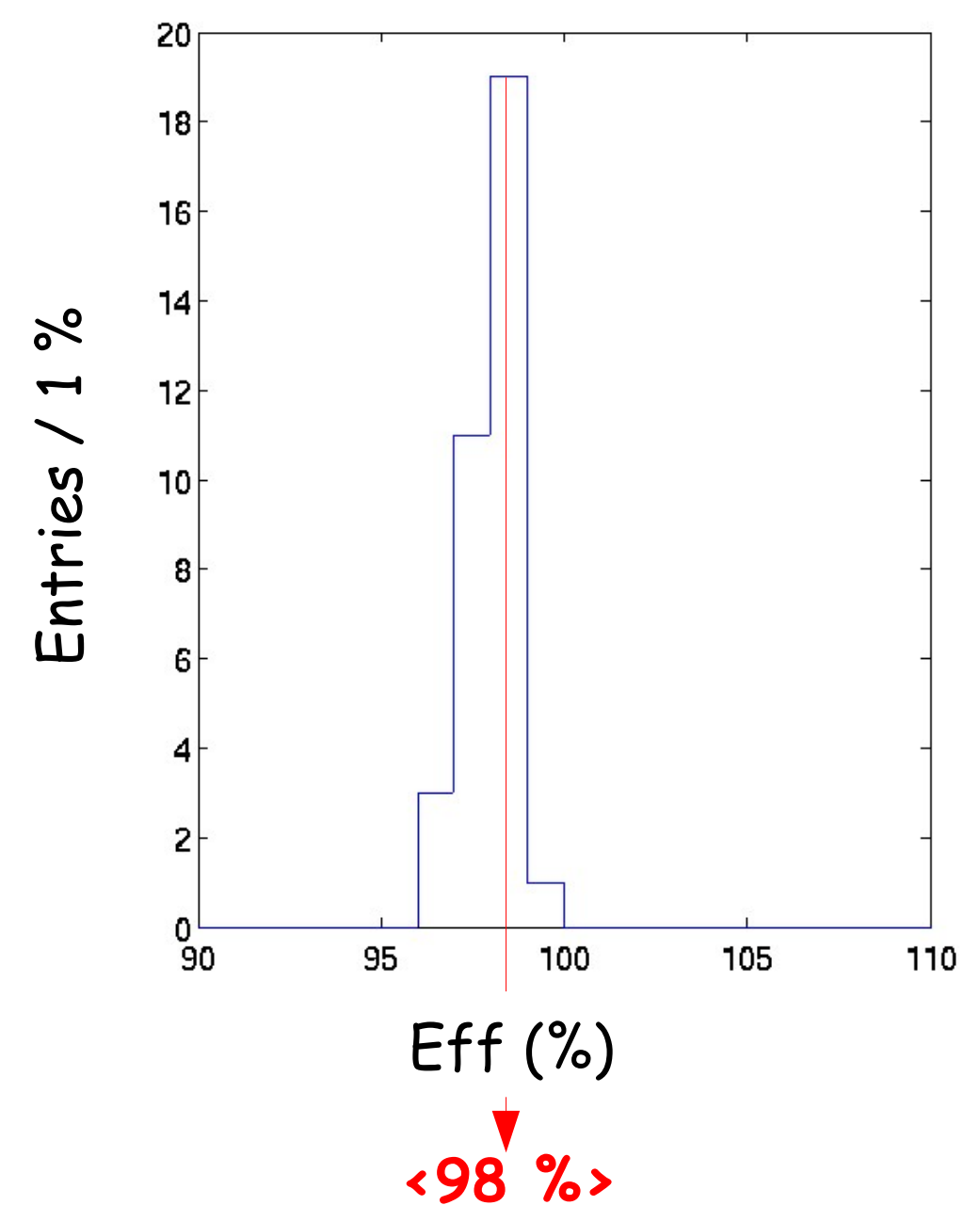

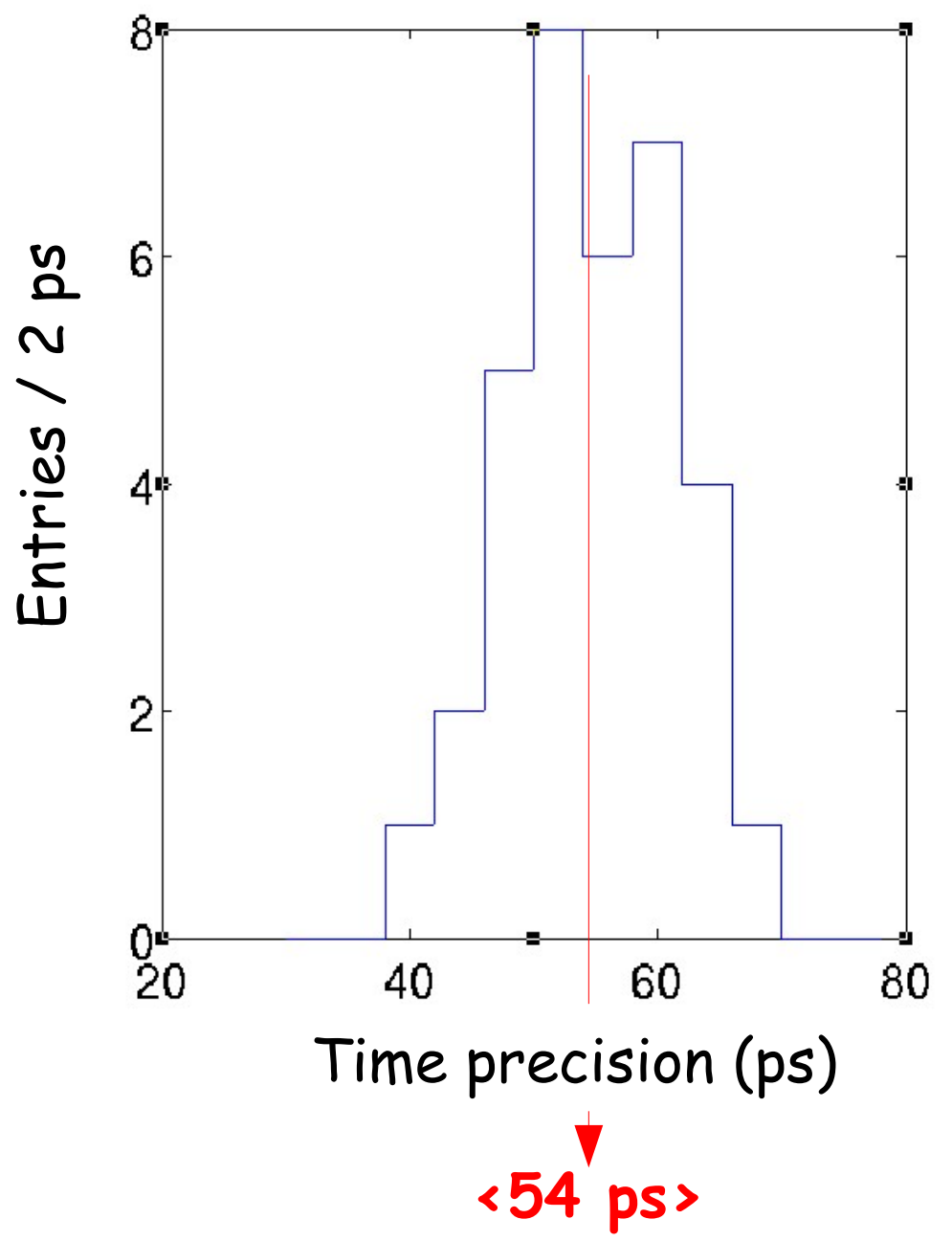


Test beam. Modifications to baseline design. Grouping strips.

Detector completely rewired during beam time

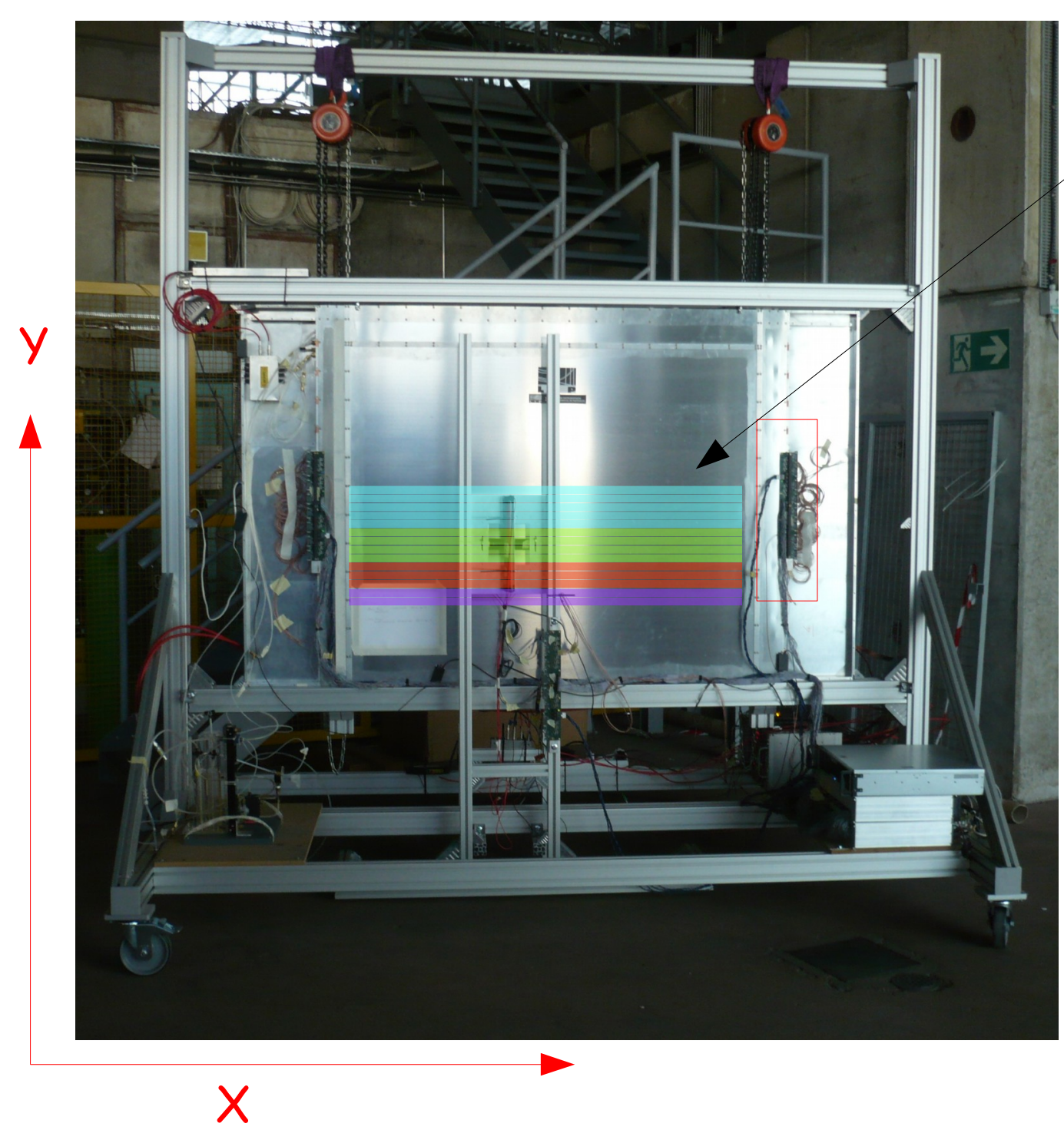

Group several strips in one single channel $2(60 \mathrm{~mm}), 3(90 \mathrm{~mm})$, $4(120 \mathrm{~mm}), 5(150 \mathrm{~mm})$ strips together

Motivation $=>$ simplify the detector, if possible, to make it cheaper but keeping performance unaltered. 
Test beam. Modifications to baseline design. Grouping strips.

Tested in the center and in one of the sides of the strips group
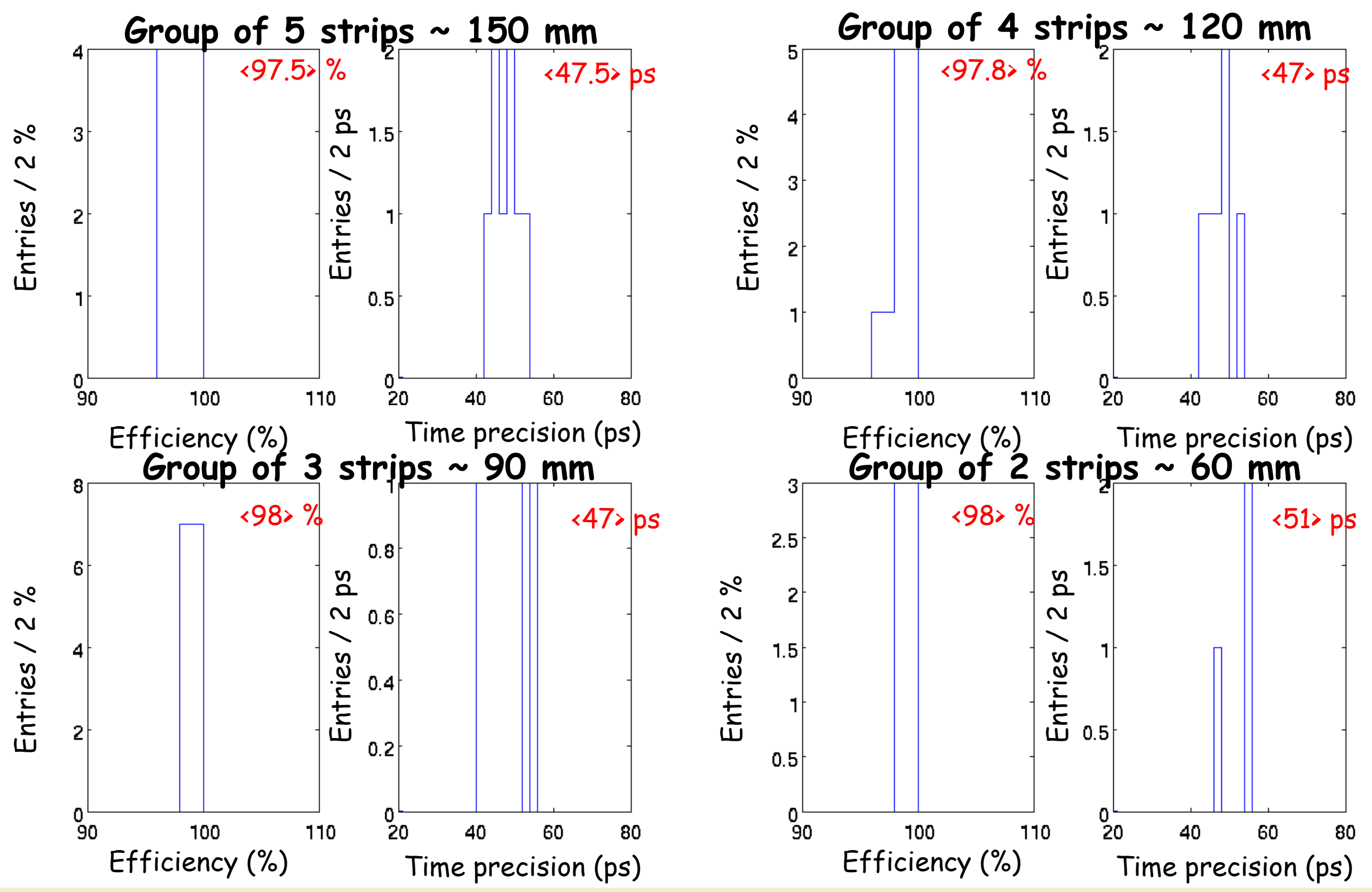
Test beam. Modifications to baseline design. One SGS.

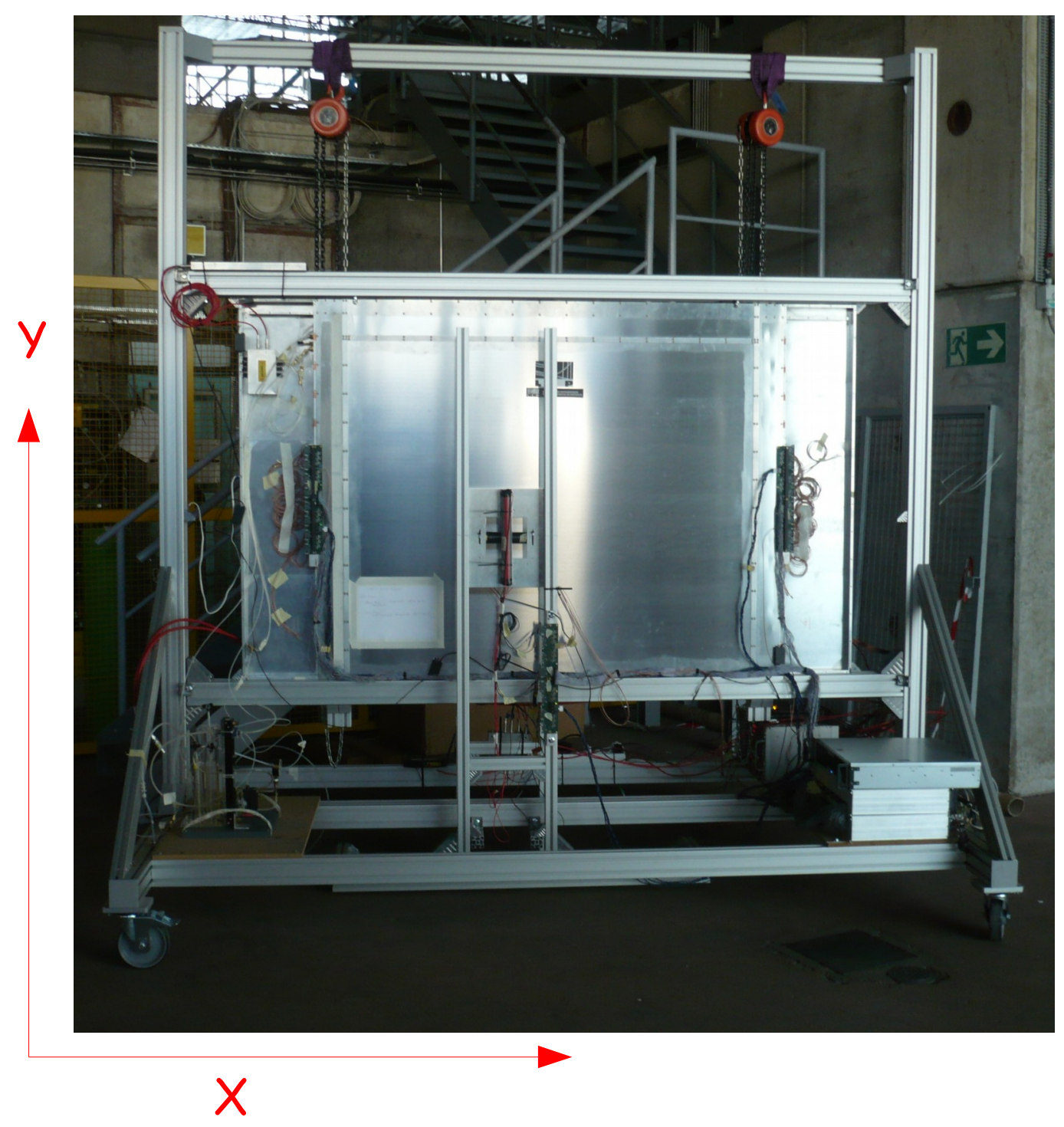

Only one glass stack connected

Motivation $=>$ simplify the detector, if possible, to make it cheaper but keeping performance unaltered. 
Test beam. Modifications to baseline design. One SGS.

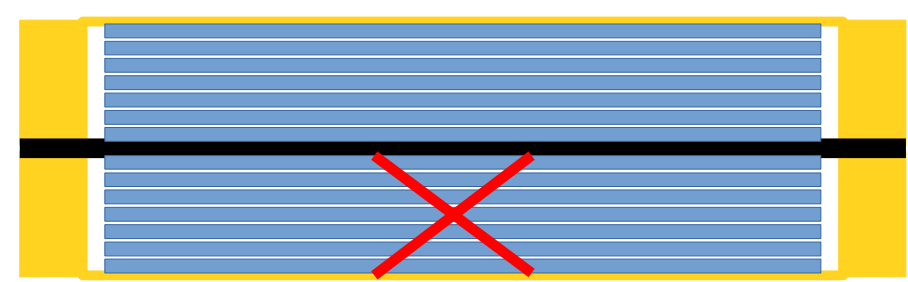

Timing precision slightly degrades but efficiency drops down to $92 \%$

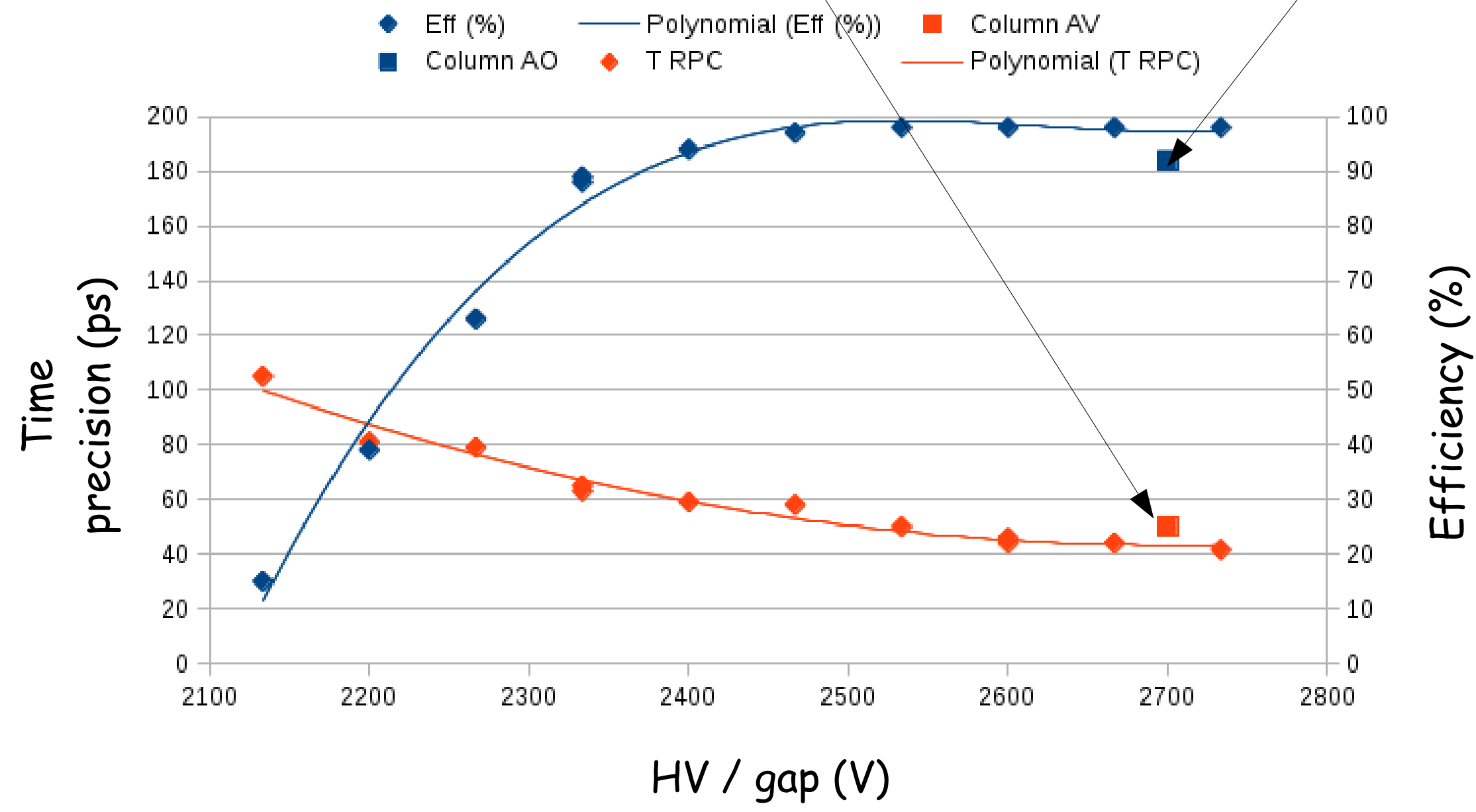




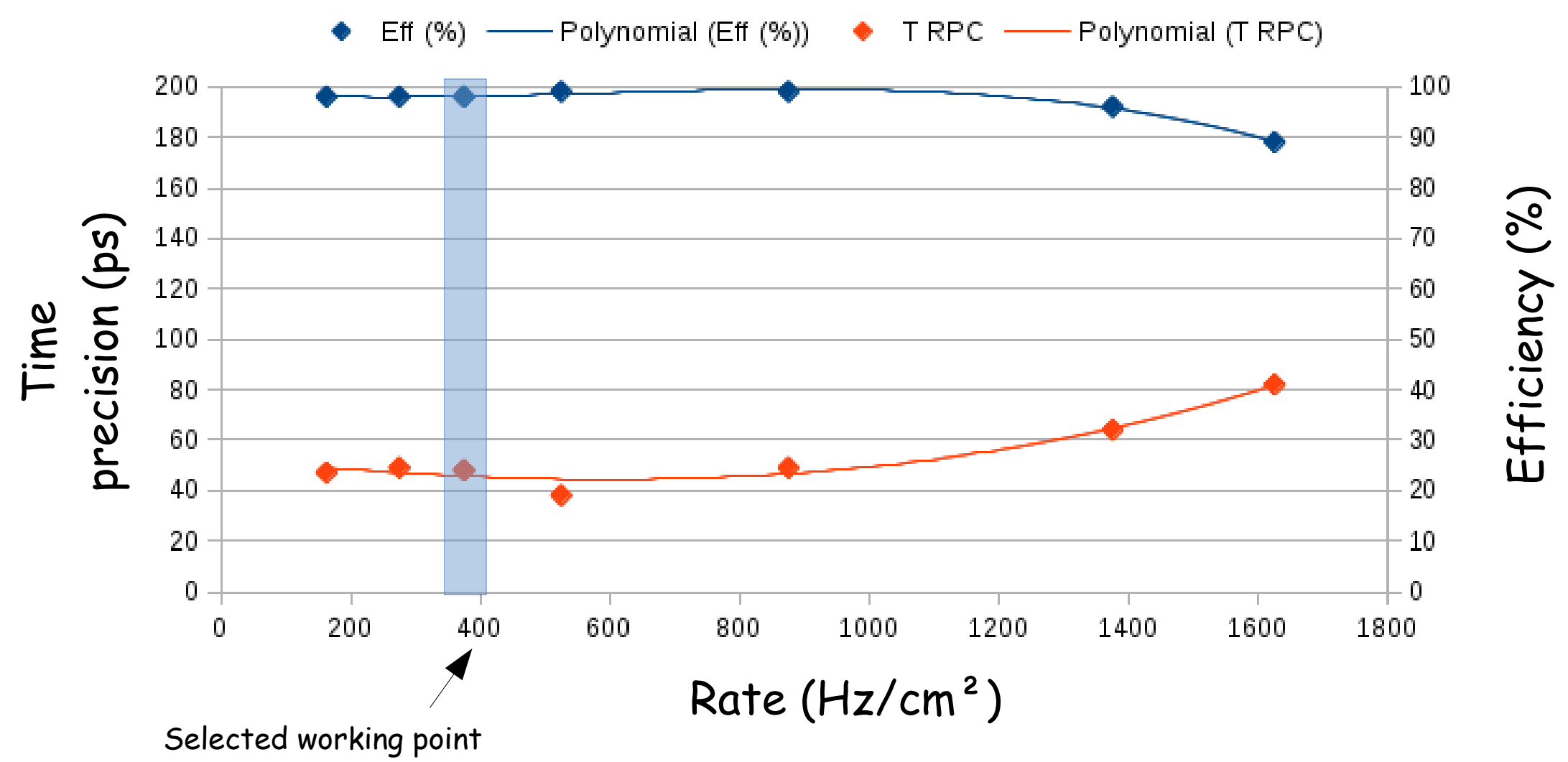

But the short spill at CERN $(0.4 \mathrm{sec})$ is quite favorable for RPC $\Rightarrow$ in continuous irradiation or spill >1-2 sec results will get worse 
Requirements of the ShiP timing detector are fulfilled by the proposed technology based on MRPC.

Timing accuracy $\sim 54$ ps together with an efficiency of $98 \%$ without noticeable dependence with position over $\sim 2 \mathrm{~m}^{2}$ active area.

Strips up to $150 \mathrm{~mm}$ width are possible without degradation.

New concept in the construction of timing RPCs that allows the construction of large area (1-2 $\left.\mathrm{m}^{2}\right)$ chambers in a easy way decreasing the production cost. 